\title{
Lifetime Comparison on Location Base Routing in Wireless Sensor Networks
}

\author{
Hadi Asharioun, Hassan Asadollahi, Abdul Samad Ismail, and Sureswaran Ramadass
}

\begin{abstract}
Lifetime of a sensor network is defined as the time after which certain fraction of sensor nodes run out of their batteries so that the network cannot work well. Increasing life time is very important in WSNs and because of energy limitation the sensors will die and the networks cannot sense. In this paper we consider the life time of the wireless sensor network we comparison between compass routing and greedy routing in triangulation graph in wireless sensor network.
\end{abstract}

Index Terms-Life time; sensor networks, delaunay triangulation.

\section{INTRODUCTION}

There are many applications on wireless sensor networks as a monitoring and tracing [1]. While various energy efficient protocols have been proposed to prolong network lifetime, lifetime analysis is notoriously difficult since the network lifetime depends on many factors including network architecture and protocols, data collection initiation, lifetime definition, channel characteristics, and energy consumption model. Upper bounds on lifetime are thus derived for various WSNs [2]

Several localized routing protocols guarantee the delivery of the packets when the underlying network topology is a planar graph[3-10]. Typically, relative neighbourhood graph (RNG) or Gabriel graph (GG) is used as such planar structure. However, it is well-known that the spanning ratios of these two graphs are not bounded by any constant (even for uniform randomly distributed points). Bose et al [10]. Recently developed a localized routing protocol that guarantees that the distance travelled by the packets is within a constant factor of the minimum if Delaunay triangulation of all wireless nodes is used, in addition, to guarantee the delivery of the packets. However, it is expensive to construct the Delaunay triangulation in a distributed manner. Given a set of wireless

Nodes, we model the network as a unit-disk graph (UDG), in which a link uv exists only if the distance $\|u v\|$ is at most the maximum transmission range. In this paper, we present a novel localized networking protocol that constructs a planar 2.5-spanner of UDG, called the localized Delaunay triangulation (LDEL), as network topology. It contains all

Manuscript received March 1, 2012; revised April 3, 2012. This work was supported inNational AdvancedIPv6 Centre (NAv6), University Science Malaysia.

Hadi Asharioun and Sureswaran Ramadass are with the National Advanced IPv6 Centre (Nav6), University Science Malaysia (e-mail: (hadi, sures)@nav6.usm.my).

Hassan Asadollahi and Abdul Samad Ismail are with the Department of Computer System and Communication, Faculty of Computer Science and Information, University Technology Malaysia (e-mail: hasan_asadolahi@yahoo.com,abdsamad@utm.my). edges that are both in the unit-disk graph and the Delaunay triangulation of all nodes. The total communication cost of our networking protocol is $\mathrm{O}(\mathrm{n} \log \mathrm{n})$ bits, which is within a constant factor of the optimum to construct any structure in a distributed manner. Our experiments show that the delivery rates of some of the existing localized routing protocols are increased when localized Delaunay triangulation is used instead of several [6]

Previously proposed topologies. Our simulations also show that the travelled distance of the packets is significantly less when the FACE routing algorithm is applied on LDEL, rather than applied on GG.

The paper is organized as follows: Section 2 discusses about different geometrical routing algorithm. In Section 3, we discuss about lifetime in compass routing and we present the result of our simulation. Finally, Section 4 gives concluding remarks. . Greedy forward routing (abbreviated by GFR) is one of the localized geographic routing algorithms proposed in literature. In GFR, one node discards a packet if none of its neighbours is closer to the destination of the packet than itself, or otherwise forwards the packet to the neighbor closest to the destination. Therefore, each packet should contain the location of its destination, and each node only needs to maintain the locations of its one-hop neighbors. GFR can be implemented in a localized and memory less manner. There are some variations of GFR. For example, in [11] and[12], the shortest projected distance to the destination on the straight line joining the current node and the destination node is considered as the greedy metrics. In [11] , packets are allowed to be sent backward if there is no forwarding neighbor. In [12], only nodes whose Voronoi cells intersect with the source destination line segment are eligible for being relay nodes. Here the Voronoi cell of a node is the set of points in the plane that are closer to the node than to any other node[13]. The analytic work of GFR can be dated back to 1984 by Takagi and Kleinrock[11]. They studied the optimal transmission radius to maximize the expected progress of packets based on most forward and least backward routing strategy in which every node delivers each packet to the neighbor (not including itself) with the shortest projected distance to the destination on the straight line joining the current node. However, the deliverability of packets is not considered. Recently, Xing et al. [12] (2004) show that in a fully covered homogeneous wireless sensor network, if the transmission radius is larger than 2 times of the sensing radius, the deliverability can be guaranteed between any source-destination pair by greedy forwarding schemes in which a packet is sent to the neighbour either with the shortest Euclidean distance to the destination[14, 15] or with the shortest projected distance to the destination on the straight line joining the current node and the destination node 
[11] and by bounded Voronoi greedy forwarding scheme in which only those nodes whose Voronoi cells intersect with the line segment between the source and destination are eligible to relay the packet. Another related and interesting problem in literature is the longest edge of connected geometric graphs. Penrose[16] (1997)[17](1999) studied the longest edge of a minimal spanning tree which is corresponding to

the critical transmission radius for connectivity in random geometric graphs. Later, by applying the percolation theory, Gupta and Kumar[18]had similar results for wireless networks. Recently, Baccelli and Bordenave[19] (2007) introduced a structure called radial spanning trees (RSTs) in which each node, excluding the root $s$ at the origin of the plane, has an edge to its closest neighbor among nodes closer to the root s. In this paper we focus on greedy algorithm and estimate lifetime on it.

\section{ROUTING Algorithms}

There are many kind of graph routing in geometrical routing as

Compass rout Compass Routing $(\mathrm{Cmp})$ : Let $\mathrm{t}$ be the destination node. Current node $\mathrm{u}$ finds the next relay node $\mathrm{v}$ such that the angle $<v u t$ is the smallest among all neighbors of $\mathrm{u}$ in a given topology. See [3].

Random Compass Routing (RndCmp): Let $\mathrm{u}$ be the current node and $t$ be the destination node. Let $v_{1}$ be the node on the above of lineut such that $\angle v_{1} u t$ is the smallest among all such neighbours ofu. Similarly, we define $v_{2}$ to be nodes below line ut that minimizes the angle $\angle v_{2} u t$. Then, node $u$ randomly chooses $v_{1}$ or $v_{2}$ to forward the packet. See [3].

Greedy Routing (Grdy): Let $\mathrm{t}$ be the destination node. Current node $\mathrm{u}$ finds the next relay node $\mathrm{v}$ such that the distance $\|v t\|$ is the smallest among all neighbours of $u$ in a given topology. See [11].

Most Forwarding Routing (MFR): Current node $u$ finds the next relay node $v$ such that $\left\|v^{\prime} t\right\|$ is the smallest among all neighbors of $\mathrm{u}$ in a given topology, where $\mathrm{v}^{\prime}$ is the projection of v on segment ut. See [7].

Nearest Neighbor Routing $(\mathrm{NN})$ : Given a parameter angle $\alpha$ node $\mathrm{u}$ finds the nearest node $\mathrm{v}$ as forwarding node among all neighbors of $\mathrm{u}$ in a given topology such that $\angle$ vut $\leq \alpha$.

Farthest Neighbor Routing (FN): Given a parameter angle $\alpha$, node $\mathrm{u}$ finds the farthest node $\mathrm{v}$ as forwarding node among all neighbors of $\mathrm{u}$ in a given topology such that $\angle \mathrm{vut} \leq \alpha$.

\section{Routing Algorithms in Planar Network TOPOLOGIES}

There are many routing algorithm on the various planar network topologies (see fig 1) [19]as a Compass rout Compass Routing (Cmp) [3], Random Compass Routing (RndCmp) [3], Most Forwarding Routing (MFR) [20], Nearest Neighbor Routing (NN), Farthest Neighbor Routing (FN) and Greedy Routing (Grdy) definition of greedy routing is: let $t$ be the destination node. Current node $u$ finds the next relay node $v$ such that the distance $v t$ is the smallest among all neighbours of $u$ in a given topology. See [20]. The mentioned routing can run on the some different graph as relative neighbourhood graph, Yao graph, Delaunay triangulation and so on (see fig 1). The mentioned graph is planner graph. In graph theory, a planner graph is a graph that can be embedded in the plane (i.e. it can be drawn on the plane in such a way that its edges intersect only at their endpoints). In other words, it can be drawn in such a way that no edges cross each other.

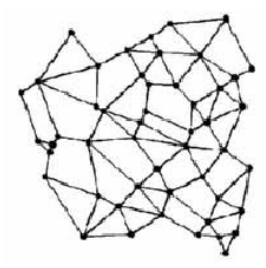

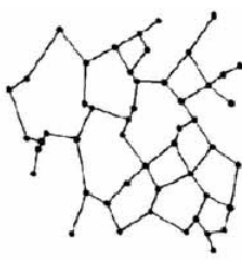

GG graph
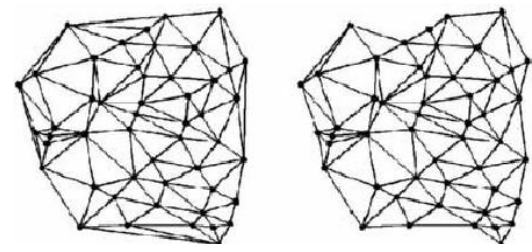

Del graph

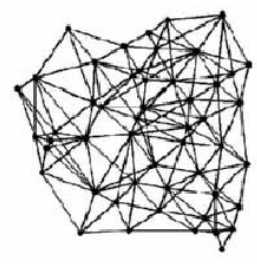

RNG graph

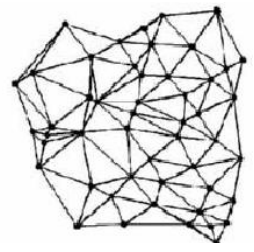

LDel graph
Fig. 1. various planar network topologies

We are focusing on the greedy routing on the random planner graph. Due to existence of local minima where none of neighbours is closer to the destination than the current node, a packet may be discarded before arriving its destination. To ensure that every packet can arrive its destination, all nodes should have sufficiently large transmission radii to avoid being local minima [21]. For points $\mathrm{x}, \mathrm{y} \in \mathbb{R} 2$ and a positive real number $\mathrm{r}$, let $\mathrm{B}(\mathrm{x}, \mathrm{r})$ denote the open disk of radius $r$ centered at $\mathrm{x},\|\mathrm{x}\|$ denote the Euclidean norm of $\mathrm{x}$, and $\|\mathrm{x}-\mathrm{y}\|$ denote the Euclidean distance betweenx and y. Consider Fig. 2. Let u be a source or relay GG graph RNG graph Yao graph PLDel graph LDel graph Del graph node, $v$ be the corresponding destination node, and $\mathrm{w}_{\mathrm{i}}$ denote nodes other than $\mathrm{u}$ and $\mathrm{v}$. Nodes that can relay packets for $\mathrm{u}$ toward $v$ must be in the region $\mathrm{B}(\mathrm{u}, \| \mathrm{u}-$ $\mathrm{v}) \cap \mathrm{B}(\mathrm{v}, \mathrm{u}-\mathrm{v})$ based on the following observations. If wi can relay packets for $u$ toward $v$, it must be closer to $v$ than $u$, i.e. $\left\|\mathrm{u}-\mathrm{w}_{\mathrm{i}}\right\|<\|\mathrm{u}-\mathrm{v}\|$ or equivalently $\mathrm{w}_{\mathrm{i}} \in \mathrm{B}(\mathrm{v},\|\mathrm{u}-\mathrm{v}\|)$. $\mathrm{w}_{2}, \mathrm{w}_{3}, \mathrm{w}_{4}$ satisfy this rule and $\mathrm{w}_{1}$ does not. On the other hand, if no one can relay packets for $u$, packets should be directly transmitted from $\mathrm{u}$ to $\mathrm{v}$. So, in the worst case, $\mathrm{u}$ at most needs to set its transmission radius to $\|\mathrm{u}-\mathrm{v}\|$.

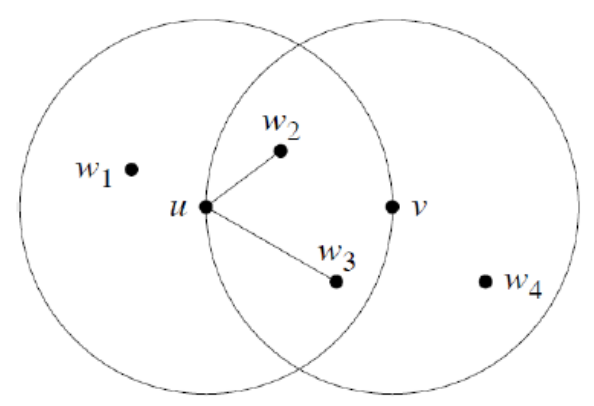

Fig. 2. $\mathrm{u}$ is a source node and $\mathrm{v}$ is the corresponding destination node.

This implies candidates of relay nodes must be in 
B $(u,\|u-v\|)[12]$.For example, in Fig. 2, $w_{4}$ can't be a candidate of relay nodes. Thus, only $\mathrm{w}_{2}$ and $\mathrm{w}_{3}$ can relay packets for utowardv. In addition, if the transmission radius is set to $\min \left(\left\|w_{2}-v\right\|,\left\|w_{3}-v\right\|\right)$, u has at least one neighbor to relay packets. The procedure of selecting the minimal transmission radii to ensure either $u$ can send packets directly to $v$ or there exists at least one node to relay packets for $\mathrm{u}$ toward $\mathrm{v}$ can be expressed as $\min \left(\mathrm{w}_{\mathrm{i}} \in\right.$ $B(v,\|u-v\|)\left\|w_{i}-u\right\|$. In the next section we want to consider the life time in by greedy routing on a planer graph.

\section{DELAUnAy TRIANGULATION}

The Delaunay triangulation $\mathrm{D}\left(P_{n}\right)$ of a set of $n$ points $P_{n}$ on the plane, is the partitioning of the convex hull of $P_{n}$ into a set of triangles with disjoint interiors such that

- the vertices of these triangles are points in $P_{n}$

- for each triangle in our triangulation the circle passing through its vertices contains no other point of $P_{n}$ in its interior.

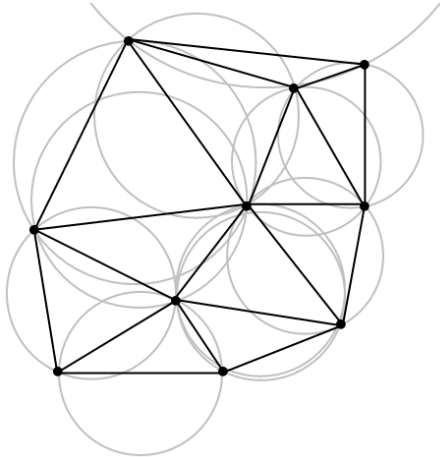

Fig. 3. A delaunay triangulation graph

\section{COMPASS ROUTING II}

We now obtain a local information routing algorithm that guarantees that any message will eventually reach its destination. We describe our algorithm first for the case in which our geometric graphs are convexly embedded, i.e. all the faces of our geometric graph are convex, except for the unbounded one which is assumed to be the complement of a convex polygon, see Figure 3. Our algorithm proceeds as follows:

Compass Routing II:

1) Starting at $\mathrm{s}$ determine the face $\mathrm{F}=\mathrm{F}_{0}$ incident to $\mathrm{s}$ intersected by the line segment st joining $s$ to $t$. Pick any of the two edges of $F_{0}$ incident to $s$, and start traversing the edges of $\mathrm{S}_{0}$ until we find the second edge, say $\mathrm{u}-\mathrm{v}$ on the boundary of $\mathrm{F}_{0}$ intersected by st.

2) At this point, we update $F$ to be the second face of our geometric graph containing $\mathrm{u}-\mathrm{v}$ on its boundary. We now traverse the edges of our new $\mathrm{F}$ until we find a second edge $\mathrm{x}-\mathrm{y}$ intersected by st. At this point we update $\mathrm{F}$ again as in the previous point. We iterate our current step until we reach $\mathrm{t}$.

Let $F_{0}, F_{1}, \ldots F_{r}$ be the faces intersected by st. Observe $F=F_{0}$ that initially $F_{0}$, and that each time we update $F$, we change its value from $F_{i}$ to $F_{i+1}$, so eventually we will reach $F_{k}$, the face containing $t$, and when we traverse its boundary we will arrive at $\mathrm{t}[3]$.

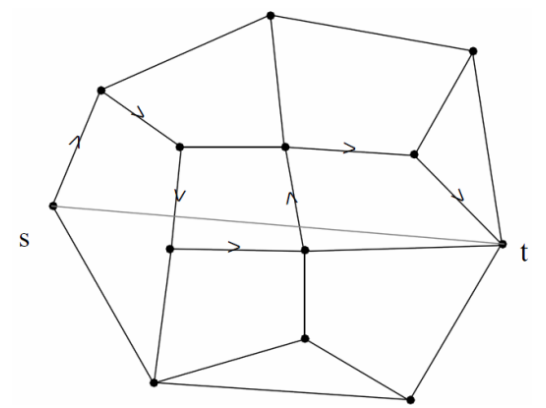

Fig. 4. Routing in convexly embedded geometricgraphs.

Network lifetime has become the key characteristic for evaluating sensor networks in an application specific way. Especially the availability of nodes, the sensor coverage, and the connectivity have been included in discussions on network lifetime. Even quality of service measures can be reduced to lifetime considerations. A great number of algorithms and methods were proposed to increase the lifetime of a sensor network while their evaluations were always based on a particular definition of network lifetime. Motivated by the great differences in existing definitions of sensor network lifetime that are used in relevant publications, we reviewed the state of the art in lifetime definitions, their differences, advantages, and limitations.

This paper was the starting point for our work towards a generic definition of sensor network lifetime for use in analytic evaluations as well as in simulation models. Focusing on a formal and concise definition of accumulated network lifetime and total network lifetime. Our definition incorporates the components of existing lifetime definitions, and introduces some additional measures. One new concept is the ability to express the service disruption tolerance of a network. Another new concept is the notion of time-integration: in many cases, it is sufficient if a requirement is fulfilled over a certain period of time, instead of at every point in time. In addition, we combine coverage and connectivity to form a single requirement called connected coverage. We show that connected coverage is different from requiring non-combined coverage and connectivity. Finally, our definition also supports the concept of graceful degradation by providing means of estimating the degree of compliance with the application requirements. We demonstrate the applicability of our definition based on the surveyed lifetime definitions as well as using some example scenarios to explain the various aspectsincensing sensor network lifetime[22].

\section{Simulation}

Network lifetime is the time span from the deployment to the instant when the network is considered nonfunctional. When a network should be considered nonfunctional is, however, application-specific. It can be, for example, the instant when the first sensor dies, a percentage of sensors die, the network partitions, or the loss of coverage occurs [2].For a WSN with total non-rechargeable initial energy $\varepsilon_{0}$, the average network lifetime $\mathbb{E}[L]$, measured as the average amount of time until the network dies, is given by [2].

$\mathbb{E}[L]=\frac{\varepsilon_{0}-\mathbb{E}\left[E_{\omega}\right]}{P_{c}+\lambda \mathbb{E}\left[E_{r}\right]}$ 
where $\mathrm{Pc}$ is the constant continuous power consumption over the whole network, $\mathbb{E}\left[\mathrm{E}_{\omega}\right]$ is the expected wasted energy (i.e., the total unused energy in the network when it dies), $\lambda$ is the average sensor reporting rate defined as the number of data collections per unit time, and $\mathbb{E}\left[\mathrm{E}_{\mathrm{r}}\right]$ is the expected reporting energy consumed by all sensors in a randomly chosen data collection. $\mathrm{In}[23]$ the authors denote $\mathrm{E}_{\mathrm{rx}}$ for receive energy of signals (one bit) and $E_{t x}$ for the transmit energy for a bit in the sensors. We suppose $\mathrm{N}$ nodes are deployed in $\mathrm{M} \times \mathrm{M}$ area And node $\mathrm{S}$ want to transfer data to node D. To transfer data node $\mathrm{S}$ desspate $\mathrm{E}_{\mathrm{rx}}$ and each relays nodes received and then transferred the data (i.e. each relay node dissipate $E_{r x}+E_{t x}$ ) then node $\mathrm{D}$ just dissipate $\mathrm{E}_{\mathrm{rx}}$. As a result, if we have k relay nodes, the energy dissipation to communicate one bit from $\mathrm{S}$ to $\mathrm{D}$ is $(\mathrm{k}+1)\left(\mathrm{E}_{\mathrm{rx}}+\mathrm{E}_{\mathrm{tx}}\right)$ and For $\mathrm{P}$ consisting of $\mathrm{N}$ points, all triangulations contain $2 \mathrm{~N}-2-\mathrm{K}$ triangles, $3 \mathrm{~N}-3-\mathrm{k}$ edges [23]. $\mathrm{N}$ is number of points in $\mathrm{P}$ and $\mathrm{k}$ is number of pint in convex hull of $\mathrm{p}$.

TABLE I: THE DELIVERY RATE OF DIFFERENT LOCALIZED ROUTING METHODS ON DELAUNAY TOPOLOGIES

\begin{tabular}{l|l} 
Routing & Rate \\
\hline Compass rout Compass Routing & $100 \%$ \\
Random Compass Routing & $100 \%$ \\
Greedy Routing & $100 \%$ \\
Most Forwarding Routing & $95.2 \%$ \\
Nearest Neighbor Routing & $99.1 \%$ \\
Farthest Neighbor Routing & $92.1 \%$
\end{tabular}

We have run simulation for the greedy routing for 100 nodes in a $1 \times 1$ unit area with node transmission range 0.3 . In each time two nodes is selected randomly and a packet is routed from source to destination and source node and destination node and relay nodes increase their counter to obtain energy consumption then to use for obtaining life time. The simulator transfers a frame 50000 times between two random selected nodes on a random graph. The result shows that greedy routing is better than compass routing in the delivery ratio and in both of compass and greedy nodes are on the face of the graph, consume energy less than others, and nodes are in the center of the graph use more energy than other nodes. Each node is connected with more edge, relay more energy as result they are died sooner than others. Also the result shows delivery rate of Delaunay is $100 \%$. Comparison between other routing algorithm shows in table1.

Also, we have run simulation for the greedy routing for 30 nodes in a $1 \times 1$ unit area with node transmission range 0.3 . In each time two nodes is selected randomly and a packet is routed from source to destination and source node and destination node and relay nodes increase their counter to obtain energy consumption then to use for obtaining life time. The simulator transfers a frame 50000 times between two random selected nodes on a

TABLE II: THE DELIVERY RATE For GREEDy ROUTING METHODS ON DIFFERENT TOPOLOGIES GRAPHS.

\begin{tabular}{l|l} 
Graph & Rate \\
\hline GG graph & $99.6 \%$ \\
RNG graph & $87.5 \%$ \\
Yao graph & $100 \%$ \\
Del graph & $100 \%$ \\
LDel graph & $100 \%$ \\
PLDel graph & $100 \%$
\end{tabular}

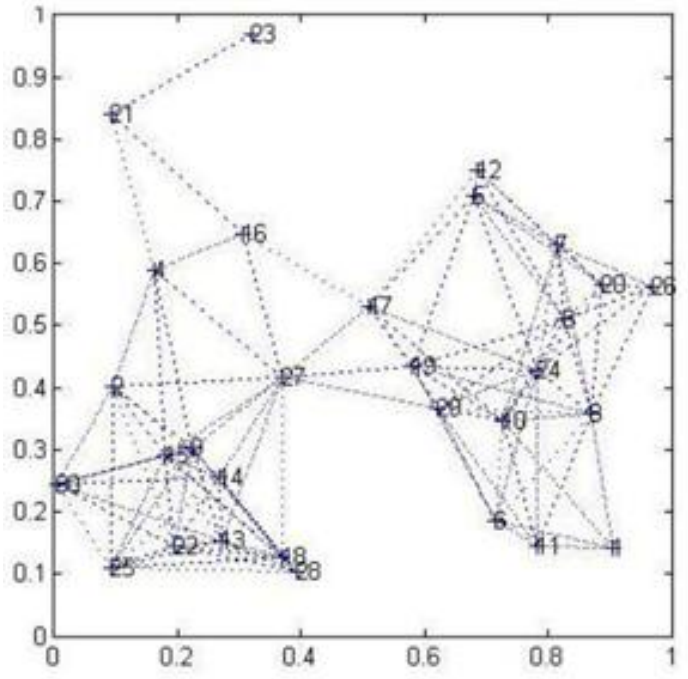

Fig. 4. A random graph 2 and number of node transfer/relay data for each node

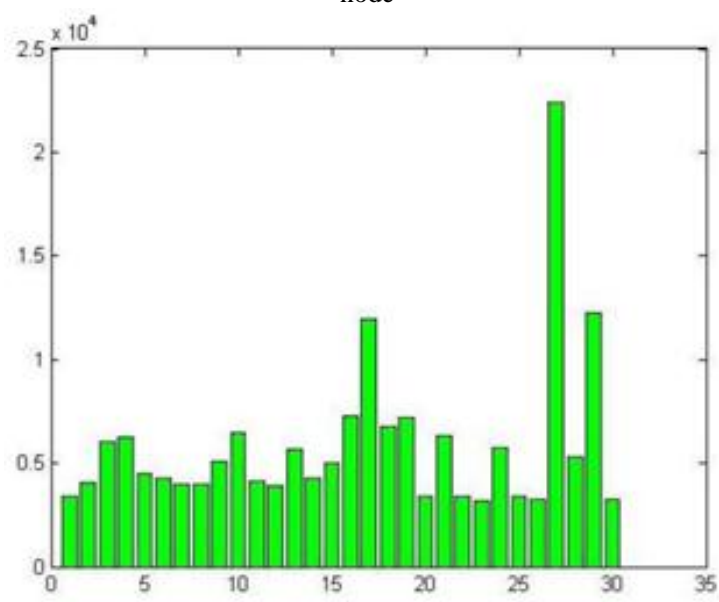

The result shows that nodes are on the face of the graph, consume energy less than others, and nodes are in the center of the graph use more energy than other nodes. Each node is connected with more edge, relay more energy as result they are died sooner than others (see Fig 3 and Fig 4). Also, the result for 100 nodes show the delivery rate for Yao graph, Del graph, LDel graph and PLDel graph is $100 \%$ (see table2).

\section{CONCLUSION}

One of the most interested open issues in wireless sensor networks is life time issue. In WSN life time is very important because the sensors have limited energy and they will die after lost their energy so that the networks cannot continue to work normally. We have considered greedy routing on the life time in the random planer graph The result shows that nodes are on the face of the graph, consume energy less than others, and nodes are in the center of the graph use more energy than other nodes. Each node is connected with more edge, relay more energy as result they are died sooner than others.

\section{REFERENCES}

[1] A. A. Abbasi and M. Younis, "A survey on clustering algorithms for wireless sensor networks," Computer Communications, vol. 30, pp. 2826-2841, 2007.

[2] Y. Chen and Q. Zhao, "On the lifetime of wireless sensor networks," Communications Letters, IEEE, vol. 9, pp. 976-978, 2005. 
[3] E. Kranakis, et al., "Compass routing on geometric networks," 1999.

[4] X. Lin and L. Stojmenovic, "Gedir: Loop-Free hybrid single-path/Flooding routing algorithm with guaranteed delivery for wireless networks," IEEE Trans. On parallel and Distr. Stst. Oct. 2001, 2001.

[5] W. Z. Song, et al., "Localized algorithms for energy efficient topology in wireless ad hoc networks," Mobile Networks and Applications, vol. 10, pp. 911-923, 2005.

[6] P.-J. W. Xiang-Yang Li, "Localized Delaunay Triangulation with Application in Ad Hoc Wireless Networks," IEEE Computer Society, 2003.

[7] I. Stojmenovic and L. Xu, "Loop-free hybrid single-path/flooding routing algorithms with guaranteed delivery for wireless networks," Parallel and Distributed Systems, IEEE Transactions on, vol. 12, pp. 1023-1032, 2001

[8] P. Bose, et al., "On the spanning ratio of gabriel graphs and B-skeletons," LATIN 2002: Theoretical Informatics, pp. 77-97, 2002.

[9] A. A. K. Jeng and R. H. Jan, "The r-neighborhood graph: An adjustable structure for topology control in wireless ad hoc networks," Parallel and Distributed Systems, IEEE Transactions on, vol. 18, pp. 536-549, 2007.

[10] P. Bose, et al., "Routing with guaranteed delivery in ad hoc wireless networks," Wireless Networks, vol. 7, pp. 609-616, 2001.

[11] H. Takagi and L. Kleinrock, "Optimal transmission ranges for randomly distributed packet radio terminals," Communications, IEEE Transactions on, vol. 32, pp. 246-257, 1984.

[12] G. Xing, et al., "On greedy geographic routing algorithms in sensing-covered networks," 2004, pp. 31-42.
[13] M. De Berg, et al., Computational geometry: algorithms and applications: Springer-Verlag New York Inc, 2008.

[14] B. Karp and H. T. Kung, "GPSR: greedy perimeter stateless routing for wireless networks," 2000, pp. 243-254.

[15] G. G. Finn, "Routing and addressing problems in large metropolitan-scale internetworks," DTIC Document 1987.

[16] M. D. Penrose, "The longest edge of the random minimal spanning tree," The annals of applied probability, vol. 7, pp. 340-361, 1997.

[17] M. D. Penrose, "A strong law for the longest edge of the minimal spanning tree," The Annals of Probability, vol. 27, pp. 246-260, 1999.

[18] P. Gupta and P. R. Kumar, "Critical power for asymptotic connectivity," 1998, pp. 1106-1110 vol. 1.

[19] X. Y. Li, et al., "Localized delaunay triangulation with application in ad hoc wireless networks," Parallel and Distributed Systems, IEEE Transactions on, vol. 14, pp. 1035-1047, 2003.

[20] I. S. a. L. Xu, "Loop-free hybrid single-path/flooding routing algorithms with guaranteed delivery for wireless networks," IEEE Transactions on, vol. 12, pp. 1023-1032, 2001

[21] P. J. Wan, et al., "Asymptotic critical transmission radii for greedy forward routing in wireless ad hoc networks," Communications, IEEE Transactions on, vol. 57, pp. 1433-1443, 2009.

[22] I. Dietrich and F. Dressler, "On the lifetime of wireless sensor networks," ACM Transactions on Sensor Networks (TOSN), vol. 5, p. 5, 2009.

[23] D. Mount, “CMSC 754: Lecture 13 Delaunay Triangulations,” 2010. 